\title{
EFFECT OF REPLACING SHARP SAND WITH STONE DUST AS FINE AGGREGATE IN CONCRETE
}

\author{
Ubi Stanley Emmanuel ${ }^{* 1}$ 不
}

${ }^{* 1}$ Department of Civil Engineering, Cross River University of Technology, Calabar

DOI: https://doi.org/10.29121/IJOEST.v5.i2.2021.176

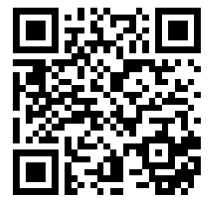

Article Type: Research Article

Article Citation: Ubi Stanley

Emmanuel. (2021). EFFECT OF

REPLACING SHARP SAND WITH

STONE DUST AS FINE AGGREGATE

IN CONCRETE. International Journal

of Engineering Science

Technologies, 5(2), 105-123.

https://doi.org/10.29121/IJOEST.v

5.i2.2021.176

Received Date: 25 March 2021

Accepted Date: 19 April 2021

Keywords:

Stone

Dust

Concrete

River

Slump

\section{ABSTRACT}

Concrete is very variable material, having a wide range of strengths and stress-strain curve. Concrete composite material whose basic properties are related to the characteristic of constituent element, especially the aggregate. This research aimed to investigate the effect of replacing sharp sand with stone dust as an alternative to only river sand. Sieve analysis was carried out on the aggregate to determine the size of particle distribution. Slump test was carried out to compare the batches of concrete for the grade, water content between batches of concrete and amount of aggregate. Compressive strength test was carried out on concrete cubes made from sharp sand and concrete cubes made from replacement of sharp sand with stone dust also concrete made from ordinary stone dust at constant of free water/cement ratio 0.5 . To check the properties of concrete produced with different materials, the tests were done for 7, 14, 21 and 28 days. The result obtained indicated that the strength of using sharp sand with stone dust in concrete production was higher than the strength of stone dust in concrete also higher than the strength of using sharp sand in concrete.

\section{INTRODUCTION}

Concrete has been considered to be very durable material requiring a little or no maintenance. The assumption is largely true, except when it is subjected to highly aggressive environments. Concrete structures are built in highly polluted urban and industrial areas, aggressive marine environments, harmful sub-soil water in coastal area and many other hostile conditions where other materials of construction are found to be non-durable (Agbede 2002., Ducatz 1995 and Ubi et al. 2020). For the past decades concrete structures have spread to highly harsh and hostile conditions, the earlier impression that concrete is a very durable material is being threatened, particularly on account of premature failure of number of structures in the recent past. Concrete is the most extensively used man made structural material. It is the product of chemical reaction between cement, sharp sand (fine aggregate), coarse aggregate and water (Fowler and Constantino 1997). The aggregate may make up to three quarters of the volume of concrete. The most durable properties of concrete are workability, high compressive strength and aesthetics. These properties are to a large degree determined by types of material used in concrete mix, especially the type and the size of aggregate. Sharp sand has been used as fine aggregate in concrete work for many years now (Nagaraji and Zahida, 1999). This is because it readily available and there has been no substitute for material. From the confined

(C) 2021 The Author(s). This is an open access article distributed under the terms of the Creative Commons Attribution License, which permits unrestricted use, distribution, and reproduction in any medium, provided the original author and source are credited. 
usefulness of concrete, there exist some proportion of limitations on the use of sharp sand with stone dust as a possible replacement for sharp sand in concrete, this is very important in view of the engineering consideration. Environmental and economic problems arising from increasing volume of stone dust accumulating and taking up space in quarry site (Owoiabi 1996). The problems of environmental degradation caused by the continuous exploitation of sand from marina sources. Hence, this paper sought to investigate the effect of replacing sharp sand with stone dust as fine aggregate in concrete. Rao et al (2002) investigated the effect of replacing sharp sand with stone dust in concrete. The tests showed strength in compression at 3 - 7 and 28 days and also strength in split tension and flexure for 7 and 28 days. The test result showed that concrete with sharp sand as fine aggregate develop a strength of $28 \mathrm{mpa}$ on the 28th day while quarry concrete at the same age developed a strength of $32.8 \mathrm{mpa}$ indicating a 17\% more compressive strength of the stone dust over the sharp and concrete (Prakash and Kumar 2004). In addition, the stone dust concrete had $7 \%$ more split tensile strength and $20 \%$ flexural strength (modules of mixture) than the concrete produce using sharp sand (Pofale and Kulkarni 1998). The result also shown that under two point loading condition, concrete with stone dust carried 6\% more load and developed smaller deflections and strains than concrete, with sharp sand with stone dust. Based on the foregoing, Rao et al (2002), of reducing the cost of concrete by $20 \%$. However, the internet has also provided many experimental facts regarding the use of quarry in concrete works reflecting the large amount of academic and industrial researches in this area. The purpose of this study research is to ascertain the current state of knowledge concerning the use of stone dust in concrete and most importantly to determine the structural and cost implication of replacing sharp sand with stone dust in concrete work and present the information in a form that would be easily understood and accessed by the student and the professional in the Civil Engineering Industry alike.

\section{MATERIALS AND METHODS}

The various materials and methods used for the research paper were adopted based on the British Standard (BS). The following materials were used: ordinary Portland cement (type 1), Fine aggregates (Sharp sand and stone dust), crushed aggregates, Sea water.

\subsection{EXPERIMENT PROCEDURES}

The grade of concrete used for the experimental project are grade $20 \mathrm{~N} / \mathrm{mm}^{2}$ and $25 \mathrm{~N} / \mathrm{mm}^{2}$. The mix design used of various grade and materials were based on weight and not on volume. Firstly, the cubes produced by sharp sand help to control the case of $20 \mathrm{~N} / \mathrm{mm}^{2}$ and $25 \mathrm{~N} / \mathrm{mm}^{2}$. Second round, the replacement of a sharp sand with stone dust as find aggregate in concrete. Thirdly, stone dust used to produce concrete cubes.

\subsection{CURING}

The purpose of curing was to control temperature and moisture into concrete, which has helped in keeping the concrete saturated as possible until the originally filled space in the fresh cement paste is occupied to the desired product of hydrated cement.

\subsection{LABORATORY TEST}

Variation test were carried out in the laboratory and this includes:

\subsection{SLUMP TEST}

Slump test is the most commonly used method of measuring consistency of concrete which can be employed in laboratory or construction site. The slump test is not a suitable method for very wet or very dry concrete. It does not measure all factors contributing to workability. However, it is used conveniently as a control test and gives an indication of the uniformity of concrete from batch to batch. Repeated batches of the same mix, brought to the same slump, will have the same water content and water cement ratio, provided the weights of the aggregate, cement and admixtures are uniform and aggregate grading is within acceptable limits. Additional information on workability 
and quality of concrete can be obtained by observing the manner in which concrete slump. Quality of concrete can also be further assessed by giving a few tapings or blows by tampering rod to the based plate. The deformation shows the characteristics of concrete with respect to tendency for segregation. The slump test gives fairly good consistent results for a plastic-mix. This test is not sensitive for a stiff-mix, in case of dry-mix, no variation can be detected between mixes of different workability. In the case of rich mixes, the value is often satisfactory, their slump being sensitive to variation in workability. IS 456 of 2000 suggests that in the "very low" category of workability where strict control is necessary for workability by determination of compacting factor will be more appropriate than slump and a value of 0.75 to 0.80 compacting factor is suggested. The bureau of Indian standards, in the past, generally adopted compacting fact or test values for denoting workability. Even in the IS 10262 of 1982 dealing with recommended guide lines for concrete mix design, adopted compacting factor for denoting workability. But now in the revision of IS 456 to 2000 the code has reverted back to slump value to denote the workability rather than compacting factor. It shows that slump test has more practical utility than the other test for workability.

\subsection{SIEVE ANALYSIS}

Sieve analysis involved dividing a sample of aggregate into various fractions, each consisting of particles of the sample size. Sieve analysis was conducted to determine the particle size distribution in a sample of aggregate, which we call gradation. A convenient system of expressing the gradation of aggregate is one which the consecutive sieve openings are constantly doubled such as $10 \mathrm{~mm}, 20 \mathrm{~mm}, 40 \mathrm{~mm}$ etc. under such a system, employing a logarithmic scale, lines can be spaced at equal intervals to represent the successive sizes. The aim of this test was to determine the range of particular size of aggregate to obtain the particle size, in each aggregate sieve analysis was carried out according to the specification in the British standard using BS 410 sieves, to know the grading for the various aggregate. The retained material in each sieve was weighed and expressed as a percentage by weight of the sample passing each of the sieves.

\subsection{COMPRESSIVE STRENGTH TEST}

About twelve cube of 150 by $150 \mathrm{~mm}$ were subjected to compressive strength test to determine their strengths for $7,14,21$, and 28 days, three cubes were crushing at the age of each day. The cube was weighed to determine the densities after weighing. The cubes were placed on the lower steel platen of the compression test machine. Compressive load was then applied at a constant rate until the sample failed for the recording according to the gauge reading at its failure point.

\subsection{CONCRETE MIX DESIGN}

This is the process of selecting suitable ingredient of concrete and determining their relative quantities with the purpose of providing an economical concrete which has certain minimum properties notably workability, strength and durability. The method used in the various mixes was British mix design. The design for the strength of 28 days of $20 \mathrm{~N} / \mathrm{mm}^{2}$ and $\mathrm{mm}^{2}$ using ordinary Portland cement with fine aggregate and coarse aggregates. $340 \mathrm{~kg} / \mathrm{mm}^{2}$ for grade 20 and $360 \mathrm{~kg} / \mathrm{mm}^{2}$ for grade 25 was cement content and the ratio of water applied in concrete production remained 0.05 .

\section{RESULTS AND DISCUSSION}

\subsection{SIEVE ANALYSIS}

\subsubsection{SHARP SAND}

Particle size distribution for the sharp sand is in the percentage shown and is presented in Table 1 and figure 1 indicating the weight at 375g:

$\begin{array}{lcc}\text { Fine } & - & 5 \% \\ \text { Medium sand } & - & 85 \% \\ \text { International Journal of Engineering } & \text { Science Technologies }\end{array}$


Effect of Replacing Sharp Sand with Stone Dust as Fine Aggregate in Concrete

Coarse sand $\quad$ - $\quad 8 \%$

Fine gravel $\quad-\quad 2 \%$

Uniformity coefficient

$\mathrm{Cu}=\frac{\mathrm{D} 60}{\mathrm{D} 10}=\frac{480 \times 10^{-3}}{250 \times 10^{-3}}=1.92$

Uniform distribution

D60 < and the particles are sandy in nature

D10

Table 1: Sieve analysis result for sharp sand total weight $=375 \mathrm{~g}$

\begin{tabular}{|c|c|c|c|c|}
\hline $\begin{array}{c}\text { B.S sieve } \\
(\mathrm{mm})\end{array}$ & $\begin{array}{c}\text { Weight retained } \\
(\mathrm{g})\end{array}$ & $\begin{array}{c}\text { Percentage retained } \\
(\%)\end{array}$ & $\begin{array}{c}\text { Percentage cumulative retained } \\
(\%)\end{array}$ & $\begin{array}{c}\text { Percentage passing } \\
(\%)\end{array}$ \\
\hline 3.35 & - & - & - & 100 \\
\hline 2.36 & 5.10 & 4.27 & 5.63 & 98.64 \\
\hline 1.18 & 16.00 & 4.27 & 5.53 & 94.37 \\
\hline 0.60 & 18.50 & 4.94 & 10.57 & 89.43 \\
\hline 0.425 & 150.50 & 40.17 & 50.74 & 49.26 \\
\hline 0.300 & 109.60 & 29.25 & 79.99 & 20.01 \\
\hline 0.212 & 58.96 & 15.72 & 95.71 & 4.29 \\
\hline 0.150 & 9.40 & 2.51 & 98.22 & 0.85 \\
\hline 0.075 & 3.50 & 0.93 & 99.15 & 0.00 \\
\hline Pan & 3.20 & 0.85 & 100.0 & \\
\hline
\end{tabular}

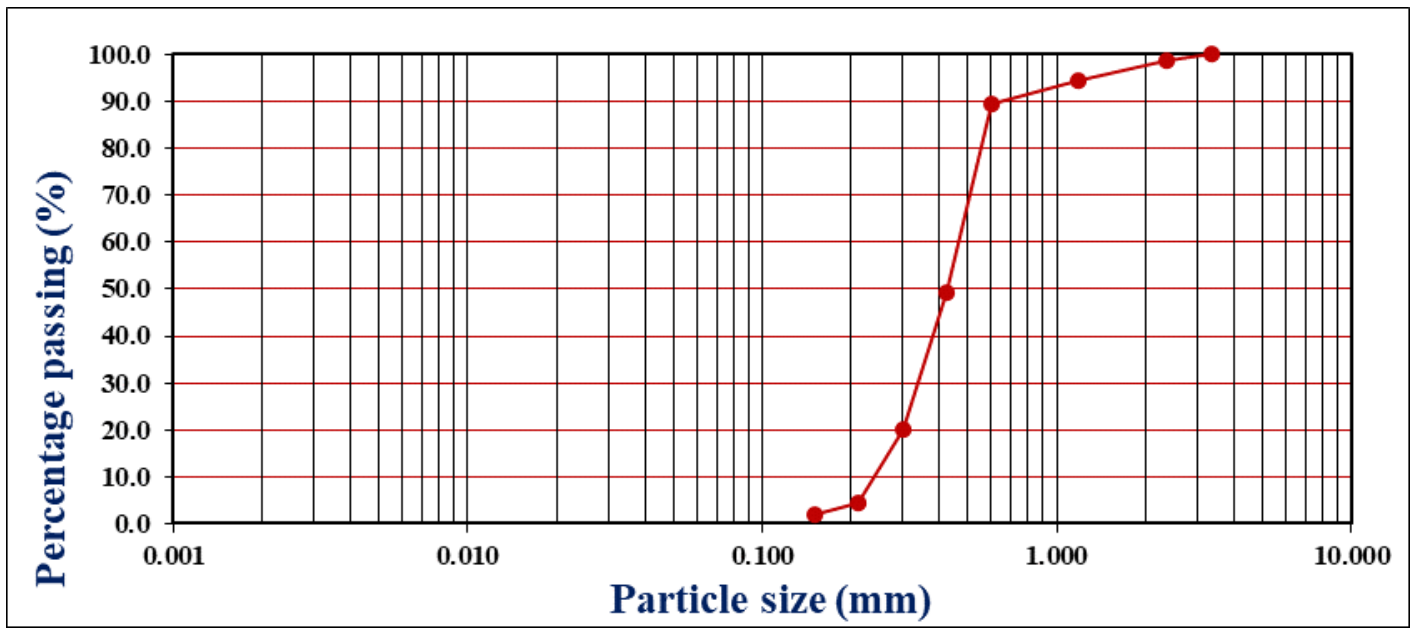

Figure 1: Sieve analysis of sharp sand

\subsection{STONE DUST}

Particle size distribution for the stone dust in percentage shown in Table 2 and figure 2 with an aggregates of 298g;

Fine $\quad-\quad 510$

Medium sand $\quad$ - $\quad 54 \%$

Coarse sand $\quad$ - $\quad 26 \%$

Fine gravel $\quad-\quad 10 \%$ 


\section{Uniformity coefficient}

$\mathrm{Cu}=\frac{\mathrm{D} 60}{\mathrm{D} 10}=\frac{599 \times 10^{-3}}{175 \times 10^{-3}}=3.42$

The particle are well grade sand with small proportion of the gravel.

Table 2: Sieve analysis of stone dust aggregates total weight - 298g

\begin{tabular}{|c|c|c|c|c|}
\hline $\begin{array}{c}\text { B.S sieve } \\
(\mathrm{mm})\end{array}$ & $\begin{array}{c}\text { Weight retained } \\
(\mathrm{g})\end{array}$ & $\begin{array}{c}\text { Percentage retained } \\
(\%)\end{array}$ & $\begin{array}{c}\text { Percentage cumulative retained } \\
(\%)\end{array}$ & $\begin{array}{c}\text { Percentage passing } \\
(\%)\end{array}$ \\
\hline 4.76 & 1.00 & 0.34 & 0.34 & 98.66 \\
\hline 2.36 & 26.70 & 8.94 & 9.28 & 90.72 \\
\hline 1.18 & 50.70 & 17.06 & 26.36 & 73.62 \\
\hline 0.60 & 22.40 & 7.52 & 32.88 & 66.10 \\
\hline 0.425 & 52.00 & 17.45 & 51.33 & 48.67 \\
\hline 0.300 & 43.10 & 14.46 & 65.79 & 32.21 \\
\hline 0.212 & 63.40 & 21.28 & 87.07 & 12.19 \\
\hline 0.150 & 12.20 & 4.09 & 91.16 & 5.82 \\
\hline 0.075 & 24.00 & 8.05 & 99.21 & 0.79 \\
\hline Pan & 2.30 & 0.79 & 100.00 & 0.00 \\
\hline
\end{tabular}

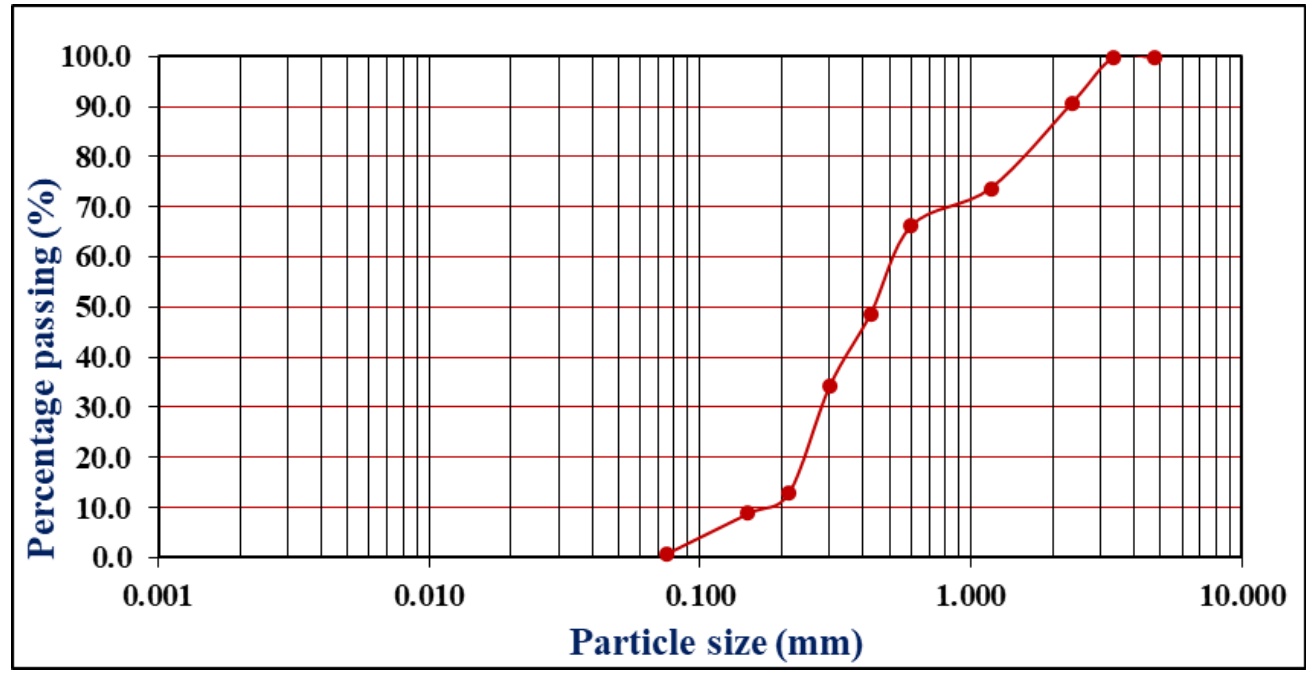

Figure 2: Sieve analysis of stone dust

\section{Coarse aggregate}

Particle size distribution shown;

Fine $\quad-\quad 8 \%$

Medium sand $\quad-\quad 8 \%$

Coarse sand $\quad$ - $\quad 7 \%$

Fine gravel $\quad-\quad 32 \%$

Medium $\quad$ - $\quad 45 \%$

\section{Uniformity coefficient}

$\mathrm{Cu}=\frac{\mathrm{D} 60}{\mathrm{D} 10}=\frac{80 \times 10^{-3}}{300 \times 10^{-3}}=2.83$

The particle is well grade sand with small proportion of the gravel as indicated in Table 3 and figure 3 with a total coarse aggregate of $1794.2 \mathrm{G}$.

International Journal of Engineering Science Technologies 
Effect of Replacing Sharp Sand with Stone Dust as Fine Aggregate in Concrete

Table 3: Sieve analysis result for coarse aggregate total weight $=1794.2 \mathrm{G}$

\begin{tabular}{|c|c|c|c|c|}
\hline $\begin{array}{c}\text { B.S Sieves Designation } \\
(\mathrm{mm})\end{array}$ & $\begin{array}{c}\text { Weight } \\
\text { retained } \\
(\mathrm{g})\end{array}$ & $\begin{array}{c}\text { Percentage } \\
\text { retained } \\
(\%)\end{array}$ & $\begin{array}{c}\text { Percentage cumulative } \\
\text { retained } \\
(\%)\end{array}$ & $\begin{array}{c}\text { Percentage } \\
\text { passing } \\
(\%)\end{array}$ \\
\hline 12.70 & 59.10 & 3.33 & 33.33 & 96.67 \\
\hline 9.50 & 335.10 & 18.68 & 22.01 & 77.99 \\
\hline 4.76 & 972.20 & 48.61 & 70.62 & 29.38 \\
\hline 2.36 & 202.50 & 11.29 & 81.91 & 18.09 \\
\hline 1.18 & 78.90 & 4.39 & 86.30 & 12.70 \\
\hline 0.60 & 22.50 & 1.25 & 87.55 & 10.68 \\
\hline 0.425 & 31.80 & 1.77 & 89.32 & 9.26 \\
\hline 0.300 & 25.50 & 1.42 & 90.74 & 7.43 \\
\hline 0.212 & 32.90 & 1.83 & 92.57 & 5.57 \\
\hline 0.150 & 33.40 & 1.86 & 94.43 & 2.99 \\
\hline 0.075 & 46.30 & 2.58 & 7.01 & 0.00 \\
\hline Pan & 53.60 & 2.99 & 100.00 & \\
\hline
\end{tabular}

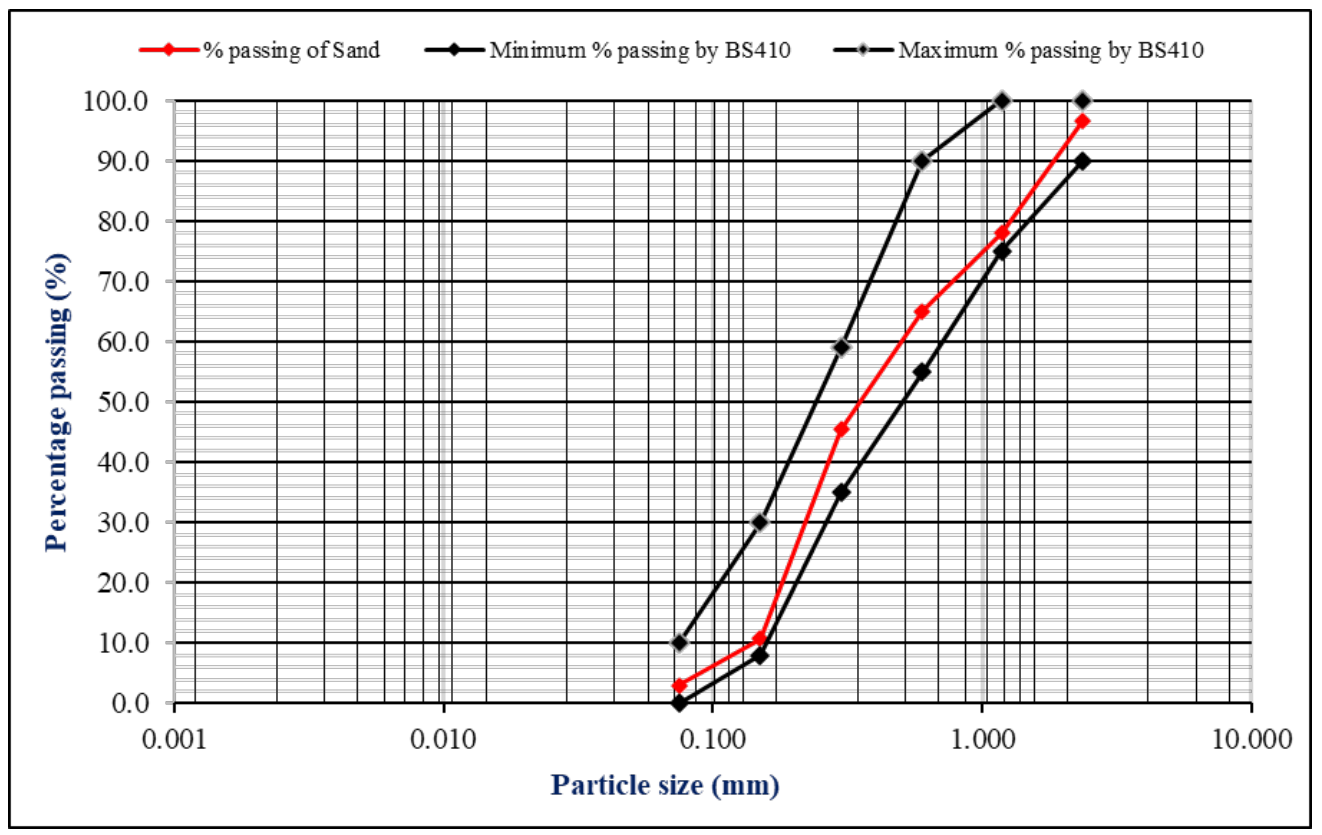

Figure 3: Sieve analysis for coarse aggregate

The British mix design method explicitly recognizes the durability requirement in the mix section. The method is applicable to normal weight of concrete made from Portland cement only. From the results obtained in Table 1-3 and figure 1 to 3 of the sieve analysis test, it is was observed that the cost of producing concrete with sharp and stone dust is almost the same. Though this title is different, can make a great deal of change in the overall cost when very large volume of concrete is required. If the site of quarry dust and if you can get the river sand close to the construction site, the price will be less. Hence, the cost of producing concrete with sharp sand with stone dust together is higher than that of river sand only.

\subsection{COMPRESSIVE STRENGTH TEST RESULT}

The strength developed by a concrete made with given material and in a given proportion increase for many months under favorable condition, but most specification put the strength at 28 days. The strength development of concrete made with all types of Portland cement depend on temperature and humidity. The strength of river sand (C20) stone dust (SDC20) and replacement of C20 with SDC 20 concrete at 7 days, 14 days, 21 days and 28 days are adequately presented in Table 4-11 respectively. 
Ubi Stanley Emmanuel

Table 4: Strength of river sand (c20) stone dust (sdc20) and replacement of C20 with SDC 20 concrete 7 day

\begin{tabular}{|c|c|c|c|c|c|c|c|c|c|}
\hline Sample No & $\begin{array}{l}\text { Date } \\
\text { Cast }\end{array}$ & $\begin{array}{l}\text { Date } \\
\text { crush }\end{array}$ & $\begin{array}{c}\text { Wt of } \\
\text { sample }(\mathrm{g})\end{array}$ & $\begin{array}{c}\text { Size of } \\
\text { sample } \\
(\mathrm{mm})\end{array}$ & $\begin{array}{l}\text { Density } \\
\mathrm{kg} / \mathrm{m}^{3}\end{array}$ & $\begin{array}{c}\text { Crushing load } \\
(\mathrm{kg})\end{array}$ & $\begin{array}{l}\text { Strength } \\
\mathrm{n} / \mathrm{mm}^{2}\end{array}$ & $\begin{array}{c}\text { Cement } \\
\text { Content } \mathrm{kg} / \mathrm{m}^{3}\end{array}$ & $\begin{array}{l}\text { Free water } \\
\text { cement ratio }\end{array}$ \\
\hline $\mathrm{C} 20 \mathrm{~A}$ & $\begin{array}{c}30-11- \\
10\end{array}$ & $7-12-10$ & 7950 & 150 & 2355 & 290 & 18.88 & 340 & 0.5 \\
\hline $\mathrm{C} 20 \mathrm{~B}$ & $\begin{array}{c}30-11- \\
10\end{array}$ & 7-12-10 & 80.12 & 150 & 2373 & 300 & 19.03. & 340 & 0.5 \\
\hline C20C & \begin{tabular}{|c|}
$30-11-$ \\
10
\end{tabular} & $7-12-10$ & 8200 & 150 & 2429 & 300 & 19.47 & 340 & 0.5 \\
\hline \multicolumn{10}{|c|}{ Average density $=2385$, average strength $=19.12$} \\
\hline \multicolumn{10}{|c|}{ SDC 20} \\
\hline SDC20A & $\begin{array}{c}30-11- \\
10\end{array}$ & $7-12-10$ & 8220 & 150 & 2435. & 310 & 1,952 & 340 & 0.5 \\
\hline SDC20B & \begin{tabular}{|c|}
$30-11-$ \\
10 \\
\end{tabular} & $7-12-10$ & 8240 & 150 & 2442 & 335 & 1957 & 340 & 0.5 \\
\hline SDC20C & \begin{tabular}{|c|}
$30-11-$ \\
10
\end{tabular} & $7-12-10$ & 7950 & 150 & 2355 & 320 & 18.88 & 340 & 0,5 \\
\hline \multicolumn{10}{|c|}{ Average density - 2410, Average strength $=19.32$} \\
\hline \multicolumn{10}{|c|}{ Replacement of C 20 with SDC 20} \\
\hline C20/SDC20A & \begin{tabular}{|c|}
$30-11-$ \\
10
\end{tabular} & $7-12-10$ & 7890 & 150 & 2337 & 220 & 18.74 & 340 & 0.5 \\
\hline C20/SDC20B & \begin{tabular}{|c|}
$30-11-$ \\
10
\end{tabular} & 7-12-10 & 8820 & 150 & 2613 & 419 & 20.94 & 340 & 0.5 \\
\hline C20/SDC20C & \begin{tabular}{|c|}
$30-11-$ \\
10
\end{tabular} & $7-12-10$ & 8300 & 150 & 2459 & 360 & 19.71 & 340 & 0.5 \\
\hline
\end{tabular}

Table 5: Strength of river sand) (C25) stone dust (SDC25) and replacement of c25 with SDC 25 concrete at 7

\begin{tabular}{|c|c|c|c|c|c|c|c|c|c|}
\hline \multicolumn{10}{|c|}{ day } \\
\hline Sample No & $\begin{array}{l}\text { Date } \\
\text { Cast }\end{array}$ & $\begin{array}{l}\text { Date } \\
\text { crush }\end{array}$ & $\begin{array}{c}\text { Wt of } \\
\text { sample (g) }\end{array}$ & $\begin{array}{c}\text { Size of } \\
\text { sample } \\
(\mathrm{mm})\end{array}$ & $\begin{array}{l}\text { Density } \\
\mathrm{kg} / \mathrm{m}^{3}\end{array}$ & $\begin{array}{l}\text { Crushing } \\
\text { load (kg) }\end{array}$ & $\begin{array}{c}\text { Strength } \\
\mathrm{n} / \mathrm{mm}^{2}\end{array}$ & $\begin{array}{c}\text { Cement } \\
\text { Content } \\
\mathrm{kg} / \mathrm{m}^{3}\end{array}$ & $\begin{array}{l}\text { Free water } \\
\text { cement ratio }\end{array}$ \\
\hline $\mathrm{C} 25 \mathrm{~A}$ & $\begin{array}{c}30- \\
11-10\end{array}$ & $\begin{array}{c}7-12- \\
10\end{array}$ & 7850 & 150 & 2357 & 280 & 18.74 & 360 & 0.5 \\
\hline $\mathrm{C} 25 \mathrm{~B}$ & $\begin{array}{c}30- \\
11-10\end{array}$ & $\begin{array}{c}7-12- \\
10\end{array}$ & 8300 & 150 & 2459 & 270 & 19.71 & 360 & 0.5 \\
\hline $\mathrm{C} 25 \mathrm{C}$ & $\begin{array}{c}30- \\
11-10\end{array}$ & $\begin{array}{c}7-12- \\
10\end{array}$ & 8360 & 150 & 2477 & 280 & 19.85 & 360 & 0.5 \\
\hline \multicolumn{10}{|c|}{ Average density $=2424$, Average strength $=19.43$} \\
\hline \multicolumn{10}{|l|}{ SDC 25} \\
\hline SDC25A & $\begin{array}{c}30- \\
11-10\end{array}$ & $\begin{array}{c}7-12- \\
10\end{array}$ & 8545 & 150 & 2531 & 330 & 1952 & 360 & 0.5 \\
\hline SDC25B & $\begin{array}{c}30- \\
11-10\end{array}$ & $\begin{array}{c}7-12- \\
10\end{array}$ & 8250 & 150 & 2442 & 250 & 1957 & 360 & 0.5 \\
\hline SDC25C & $\begin{array}{c}30- \\
11-10\end{array}$ & $\begin{array}{c}7-12- \\
10\end{array}$ & 8270 & 150 & 2450 & 320 & 18.88 & 360 & 0.5 \\
\hline \multicolumn{10}{|c|}{ Average density $=2475$, Average strength $=19.84$} \\
\hline \multicolumn{10}{|c|}{ Replacement of C 25 with SDC 25} \\
\hline $\mathrm{C} 25 / \mathrm{SDC} 25 \mathrm{~A}$ & \begin{tabular}{|c|}
$30-$ \\
$11-10$
\end{tabular} & $\begin{array}{c}-12- \\
10\end{array}$ & 7530 & 150 & 2231 & 270 & 18.74 & 360 & 0.5 \\
\hline $\mathrm{C} 25 / \mathrm{SDC} 25 \mathrm{~B}$ & $\begin{array}{c}30- \\
11-10\end{array}$ & $\begin{array}{c}7-12- \\
10\end{array}$ & 8600 & 150 & 2548 & 300 & 20.94 & 360 & 0.5 \\
\hline $\mathrm{C} 25 / \mathrm{SDC} 25 \mathrm{C}$ & $\begin{array}{c}30- \\
11-10\end{array}$ & $\begin{array}{c}7-12- \\
10\end{array}$ & 8700 & 150 & 2577 & 280 & 19.71 & 360 & 0.5 \\
\hline
\end{tabular}


Effect of Replacing Sharp Sand with Stone Dust as Fine Aggregate in Concrete

Average density $=2452$, Average strength $=19.65$

Table 6: Strength of river sand (C20) stone dust (SDC20) and replacement of c20 with SDC concrete 14 day

\begin{tabular}{|c|c|c|c|c|c|c|c|c|c|}
\hline Sample No & Cast & $\begin{array}{c}\text { Date } \\
\text { Crushed }\end{array}$ & $\begin{array}{c}\text { Wt of } \\
\text { sample (g) }\end{array}$ & $\begin{array}{l}\text { Size of } \\
\text { sample } \\
\text { (mm3) }\end{array}$ & $\begin{array}{l}\text { Density } \\
\mathrm{kg} / \mathrm{m} 3\end{array}$ & $\begin{array}{l}\text { Crushing } \\
\text { load (kg) }\end{array}$ & $\begin{array}{c}\text { Strength } \\
\mathrm{n} / \mathrm{mm} 2\end{array}$ & $\begin{array}{l}\text { Cement } \\
\text { content } \\
\mathrm{kg} / \mathrm{m} 3\end{array}$ & $\begin{array}{c}\text { Free water } \\
\text { cement ratio }\end{array}$ \\
\hline $\mathrm{C} 20 \mathrm{~A}$ & \begin{tabular}{|c|}
$30-11-$ \\
10
\end{tabular} & $\begin{array}{c}14-12- \\
10\end{array}$ & 7915 & 150 & 2345 & 460 & 18.79 & 340 & 0.5 \\
\hline C20B & \begin{tabular}{|c|}
$30-11-$ \\
10
\end{tabular} & $\begin{array}{c}14-12- \\
10\end{array}$ & 8030 & 150 & 2379 & 320 & 19,07 & 340 & 0.5 \\
\hline C20C & \begin{tabular}{|c|}
$30-11-$ \\
10
\end{tabular} & \begin{tabular}{|c|}
$14-12-$ \\
10 \\
\end{tabular} & 8040 & 150 & 2382 & 390 & 19.09 & 340 & 0.5 \\
\hline \multicolumn{10}{|c|}{ Average density $=\mathbf{2 3 6 8}$, average strength $=18.98$} \\
\hline \multicolumn{10}{|c|}{ SDC 20} \\
\hline SDC20A & \begin{tabular}{|c|}
$30-11-$ \\
10
\end{tabular} & $\begin{array}{c}14-12- \\
10\end{array}$ & 8040 & 150 & 2382 & 470 & 19.09 & 340 & 0.5 \\
\hline SDC20B & \begin{tabular}{|c|}
$30-11-$ \\
10
\end{tabular} & $\begin{array}{c}14-12- \\
10\end{array}$ & 7915 & 150 & 2345 & 300 & 18.79 & 340 & 0.5 \\
\hline SDC20C & \begin{tabular}{|c|}
$30-11-$ \\
10
\end{tabular} & $\begin{array}{c}14-12- \\
10\end{array}$ & .8030 & 150 & 2379 & 468 & 19.07 & 340 & 0.5 \\
\hline \multicolumn{10}{|c|}{ Average density $=2368$, Average strength $=18,98$} \\
\hline \multicolumn{10}{|c|}{ Replacement of C 20 with SDC 20} \\
\hline C20/SDC20A & \begin{tabular}{|c|}
$30-11-$ \\
10 \\
\end{tabular} & \begin{tabular}{|c|}
$14-12-$ \\
10 \\
\end{tabular} & 8213 & 150 & 2433 & 350 & $19: 50$ & 340 & 0.5 \\
\hline C20/SDC20B & $\begin{array}{c}30-11- \\
10 \\
\end{array}$ & $\begin{array}{c}14-12- \\
10 \\
\end{array}$ & 7817 & 150 & 2316 & 380 & 18.56 & 340 & 0.5 \\
\hline C20/SDC20C & \begin{tabular}{|c|}
$30-11-$ \\
10
\end{tabular} & $\begin{array}{c}14-12- \\
10\end{array}$ & 8200 & 150 & 2429 & 410 & 19.47 & 340 & 0.5 \\
\hline
\end{tabular}

Table 7: Strength of river sand (C25) stone dust (SDC25) and replacement of c25 with SDC 25 concreted at- 14

\begin{tabular}{|c|c|c|c|c|c|c|c|c|c|}
\hline Sample No & $\begin{array}{c}\text { Date } \\
\text { Cast }\end{array}$ & $\begin{array}{c}\text { Date } \\
\text { crush }\end{array}$ & $\begin{array}{c}\text { Wt of sample } \\
(\mathrm{g})\end{array}$ & $\begin{array}{c}\text { Size of sample } \\
(\mathrm{mm})\end{array}$ & $\begin{array}{c}\text { Density } \\
\mathrm{kg} / \mathrm{m}^{3}\end{array}$ & $\begin{array}{c}\text { Crushing } \\
\text { load } \\
(\mathrm{kg})\end{array}$ & $\begin{array}{c}\text { Strength } \\
\mathrm{n} / \mathrm{mm}\end{array}$ & $\begin{array}{c}\text { Cement } \\
\text { Content } \\
\mathrm{kg} / \mathrm{m}^{3}\end{array}$ & $\begin{array}{c}\text { Free water } \\
\text { cement ratio }\end{array}$ \\
\hline C25A & $\begin{array}{c}30-11- \\
10\end{array}$ & $\begin{array}{c}14-12- \\
10\end{array}$ & 8132 & 150 & 2409 & 300 & 19.31 & 360 & 0.5 \\
\hline C25B & $\begin{array}{c}30-11- \\
10\end{array}$ & $\begin{array}{c}14-12- \\
10\end{array}$ & $\cdot$ & $\mathrm{W}$ & 2260 & 290 & 18.12 & 360 & 0.5 \\
\hline C25C & $\begin{array}{c}30-11- \\
10\end{array}$ & $\begin{array}{c}14-12- \\
10\end{array}$ & 7850 & 150 & 2325 & 300 & 18.64 & 360 & 0.5 \\
\hline
\end{tabular}

Average density $=2331$, Average strength $=18.69$

SDC 25

\begin{tabular}{|c|c|c|c|c|c|c|c|c|c|}
\hline SDC25A & $\begin{array}{c}30-11- \\
10\end{array}$ & $\begin{array}{c}14-12- \\
10\end{array}$ & 8959 & 150 & 2651 & 375 & 21.25 & 360 & 0.5 \\
\hline SDC25B & $\begin{array}{c}30-11- \\
10\end{array}$ & $\begin{array}{c}14-12- \\
10\end{array}$ & 8850 & 150 & 2622 & 373 & 21.01 & 360 & 0.5 \\
\hline SDC25C & $\begin{array}{c}30-11- \\
10\end{array}$ & $\begin{array}{c}14-12- \\
10\end{array}$ & 8135 & 150 & 2410 & 362 & 19,22 & 360 & 0,5 \\
\hline Average density = 2561, Average strength = 20.49 \\
\hline Replacement of C 25 with SDC 25 \\
\hline $\begin{array}{c}30-11- \\
10\end{array}$ & $\begin{array}{c}14-12- \\
10\end{array}$ & 7890 & 150 & 2364 & 300 & 18.95 & 360 & 0.5 \\
\hline C25/SDC25A & $\begin{array}{c}10 \\
10-11-\end{array}$ & $\begin{array}{c}14-12- \\
10\end{array}$ & 8880 & 150 & 2631 & 340 & 21.09 & 360 & 0.5 \\
\hline
\end{tabular}


Ubi Stanley Emmanuel

\begin{tabular}{|l|c|c|c|c|c|c|c|c|c|}
\hline C25/SDC25C & $\begin{array}{c}30-11- \\
10\end{array}$ & $\begin{array}{c}14-12- \\
10\end{array}$ & 7620 & 150 & 2258 & 300 & 18.09 & 360 & 0.5 \\
\hline
\end{tabular}

Table 8: Strength of river sand (C20) stone dust (SDC20) and replacement of C20 with SDC concrete 21 day

\begin{tabular}{|c|c|c|c|c|c|c|c|c|c|}
\hline Sample No & $\begin{array}{l}\text { Date } \\
\text { cast }\end{array}$ & $\begin{array}{c}\text { Date } \\
\text { crushed }\end{array}$ & $\begin{array}{c}\text { Wt of } \\
\text { sample }(\mathrm{g})\end{array}$ & $\begin{array}{c}\text { Size of sample } \\
\text { (mm3) }\end{array}$ & $\begin{array}{l}\text { Density } \\
\mathrm{kg} / \mathrm{m} 3\end{array}$ & $\begin{array}{l}\text { Crushing } \\
\text { load (kg) }\end{array}$ & $\begin{array}{c}\text { Strength } \\
\mathrm{n} / \mathrm{mm} 2\end{array}$ & $\begin{array}{l}\text { cement } \\
\text { content } \\
\mathrm{kg} / \mathrm{m} 3\end{array}$ & $\begin{array}{c}\text { Free water } \\
\text { cement ratio }\end{array}$ \\
\hline $\mathrm{C} 20 \mathrm{~A}$ & $\begin{array}{c}30-11- \\
10\end{array}$ & $21-12-10$ & 8025 & 150 & 2377 & 400 & 19.06 & 340 & 0.5 \\
\hline $\mathrm{C} 20 \mathrm{~B}$ & \begin{tabular}{|c|}
$30-11-$ \\
10 \\
\end{tabular} & $21-12-10$ & 8887 & 150 & 2633 & 430 & 21.10 & 340 & 0.5 \\
\hline $\mathrm{C} 20 \mathrm{C}$ & \begin{tabular}{|c|}
$30-11-$ \\
10
\end{tabular} & $21-12-10$ & 8539 & 150 & 2530 & 400 & 20.28 & 340 & 0.5 \\
\hline \multicolumn{10}{|c|}{ Average density $=2313$, Average strength $=2014$} \\
\hline \multicolumn{10}{|c|}{ SDC 20} \\
\hline SDC20A & \begin{tabular}{|c|}
$30-11-$ \\
10 \\
\end{tabular} & $21-12-10$ & 8747 & 150 & 2591 & 450 & 20.77 & 340 & 0.5 \\
\hline SDC20A & \begin{tabular}{|c|}
$30-11-$ \\
10
\end{tabular} & $21-12-10$ & 8507 & 150 & 2520 & 500 & 202.0 & 340 & 0.5 \\
\hline SDC20A & \begin{tabular}{|c|}
$30-11-$ \\
10
\end{tabular} & $21-12-10$ & 8992 & 150 & 2664 & 500 & 21.35 & 340 & 0.5 \\
\hline \multicolumn{10}{|c|}{ Average density $=2916$, average strength $=20.77$} \\
\hline \multicolumn{10}{|c|}{ Replacement of C 20 With SDC 20} \\
\hline C20/SDC20A & \begin{tabular}{|c|}
$30-11-$ \\
10 \\
\end{tabular} & $21-12-10$ & 8200 & 150 & 2429 & 490 & 19.47 & 340 & 0.5 \\
\hline C20/SDC20A & $\begin{array}{c}30-11- \\
10 \\
\end{array}$ & $21-12-10$ & 8995 & 150 & 2665 & 450 & 21.36 & 340 & 0.5 \\
\hline C20/SDC20A & \begin{tabular}{|c|}
$30-11-$ \\
10
\end{tabular} & $21-12-10$ & 8600 & 150 & 2548 & 430 & 20.42 & 340 & 0.5 \\
\hline
\end{tabular}

Table 9: Strength of river sand (C25) stone dust (SDC25) and replacement of C25 with SDC 25 concrete at 21 day

\begin{tabular}{|c|c|c|c|c|c|c|c|c|c|}
\hline Sample No & $\begin{array}{l}\text { Date } \\
\text { Cast }\end{array}$ & $\begin{array}{l}\text { Date } \\
\text { crush }\end{array}$ & $\begin{array}{c}\text { Wt of } \\
\text { sample }(\mathrm{g})\end{array}$ & $\begin{array}{c}\text { Size of } \\
\text { sample }(\mathrm{mm})\end{array}$ & $\begin{array}{l}\text { Density } \\
\mathrm{kg} / \mathrm{m}^{3}\end{array}$ & $\begin{array}{l}\text { Crushing } \\
\text { load (kg) }\end{array}$ & $\begin{array}{l}\text { Strength } \\
\mathrm{n} / \mathrm{mm}^{2}\end{array}$ & $\begin{array}{c}\text { Cement } \\
\text { Content } \\
\mathrm{kg} / \mathrm{m}^{3}\end{array}$ & $\begin{array}{l}\text { Free water } \\
\text { cement ratio }\end{array}$ \\
\hline C25A & \begin{tabular}{c|}
$30-11-$ \\
10
\end{tabular} & $\begin{array}{c}21-12- \\
10\end{array}$ & 7835 & 150 & 2321 & 340 & 18.60 & 360 & 0.5 \\
\hline C25B & \begin{tabular}{c|}
$30-11-$ \\
10
\end{tabular} & $\begin{array}{c}21-12- \\
10\end{array}$ & 8100 & 150 & 2400 & 400 & 19.23 & 360 & 0.5 \\
\hline $\mathrm{C} 25 \mathrm{C}$ & $\begin{array}{c}30-11- \\
10\end{array}$ & $\begin{array}{c}21-12- \\
10\end{array}$ & 7640 & 150 & 2263 & 390 & 18.14 & 360 & 0.5 \\
\hline \multicolumn{10}{|c|}{ Average density $=2328$, Average strength $=18.65$} \\
\hline \multicolumn{10}{|c|}{ SDC 25} \\
\hline SDC25A & \begin{tabular}{|c|}
$30-11-$ \\
10
\end{tabular} & $\begin{array}{c}21-12- \\
10\end{array}$ & g365v & 150 & 2378 & 450 & 19.86 & 360 & 0.5 \\
\hline SDC25B & $\begin{array}{c}30-11- \\
10\end{array}$ & $\begin{array}{c}21-12- \\
10\end{array}$ & 7890 & 150 & 2338 & 495 & 18.73 & 360 & 0.5 \\
\hline SDC25C & \begin{tabular}{|c|}
$30-11-$ \\
10
\end{tabular} & $\begin{array}{c}21-12- \\
10\end{array}$ & & 150 & 2596 & 500 & 20.81 & 360 & 0.5 \\
\hline \multirow{2}{*}{\multicolumn{10}{|c|}{$\begin{array}{l}\text { Average density }=2440 \text {, Average strength }=19.08 \\
\text { Replacement of C } 25 \text { with SDC } 25\end{array}$}} \\
\hline & & & \multicolumn{7}{|c|}{ Replacement of C 25 with SDC 25} \\
\hline C25/SDC25A & \begin{tabular}{c|c|}
$30-11-$ \\
10
\end{tabular} & $\begin{array}{c}21-12- \\
10\end{array}$ & 8539 & 150 & 2530 & 400 & 20.28 & 360 & 0.5 \\
\hline
\end{tabular}


Effect of Replacing Sharp Sand with Stone Dust as Fine Aggregate in Concrete

\begin{tabular}{|l|c|c|c|c|c|c|c|c|c|}
\hline C20/SDC25B & $\begin{array}{c}30-11- \\
10\end{array}$ & $\begin{array}{c}21-12- \\
10\end{array}$ & 7743 & 150 & 2294 & 360 & 18.39 & 360 & 0.5 \\
\hline C20/SDC2C & $\begin{array}{c}30-11- \\
10\end{array}$ & $\begin{array}{c}21-12- \\
10\end{array}$ & 8887 & 150 & 2633 & 440 & 21.10 & 360 & 0.5 \\
\hline
\end{tabular}

Table 10: Strength of river sand (C20) stone dust (SDC20) and replacement of C20 with SDC 20 concrete 28 day

\begin{tabular}{|c|c|c|c|c|c|c|c|c|c|}
\hline Sample No & $\begin{array}{l}\text { Date } \\
\text { Cast }\end{array}$ & $\begin{array}{c}\text { Date } \\
\text { crushed }\end{array}$ & $\begin{array}{c}\text { Wt of } \\
\text { sample (g) }\end{array}$ & $\begin{array}{l}\text { Size of } \\
\text { sample } \\
\text { (mm3) }\end{array}$ & $\begin{array}{l}\text { Density } \\
\mathrm{kg} / \mathrm{m}^{3}\end{array}$ & \begin{tabular}{|c|} 
Crushing load \\
$(\mathrm{kg})$
\end{tabular} & $\begin{array}{c}\text { Strength } \\
\mathrm{n} / \mathrm{mm}\end{array}$ & $\begin{array}{l}\text { Cement } \\
\text { content } \\
\mathrm{kg} / \mathrm{m}^{3}\end{array}$ & $\begin{array}{c}\text { Free water } \\
\text { cement ratio }\end{array}$ \\
\hline $\mathrm{C} 20 \mathrm{~A}$ & $\begin{array}{c}30-11- \\
10\end{array}$ & $28-12-10$ & 8580 & 150 & 2542 & 510 & 20.39 & 340 & 0.5 \\
\hline C20B & $\begin{array}{c}30-11- \\
10\end{array}$ & $28-12-10$ & 8630 & 150 & 2557 & 520 & 20.40 & 340 & 0.5 \\
\hline C2Q.C & $\begin{array}{c}30-11- \\
10\end{array}$ & $28-12-10$ & 8430 & 150 & 2497 & 500 & 20.02 & 340 & 0.5 \\
\hline \multicolumn{10}{|c|}{ Average density $=2532$, Average strength $=20.29$} \\
\hline \multicolumn{10}{|c|}{ SDC 20} \\
\hline SDC20A & $\begin{array}{c}30-11- \\
10\end{array}$ & $28-12-10$ & 8600 & 150 & 2548 & 610 & 20.42 & & 0.5 \\
\hline SDC20A & $\begin{array}{c}30-11- \\
10\end{array}$ & $28-12-10$ & 8730 & 150 & 2586 & 620 & 20.73 & 340 & 0.5 \\
\hline SDC20A & $\begin{array}{c}30-11- \\
10\end{array}$ & $28-12-10$ & 8700 & 150 & 2577 & 610 & 20.66 & 340 & 0.5 \\
\hline \multicolumn{10}{|c|}{ Average density $=\mathbf{2 5 7 0}$, Average strength. $=\mathbf{2 0 . 6 0}$} \\
\hline \multicolumn{10}{|c|}{ Replacement of C 20 with SDC 20} \\
\hline C20/SDC20A & $\begin{array}{c}30-11- \\
10\end{array}$ & $28-12-10$ & 8500 & 150 & 2518 & 520 & 20.18 & 340 & 0.5 \\
\hline C20/SDC20B & $\begin{array}{c}30-11- \\
10\end{array}$ & $28-12-10$ & 8635 & 150 & 2558 & 530 & 20.50 & 340 & 0.5 \\
\hline C20/SDC20C & $\begin{array}{c}30-11- \\
10\end{array}$ & $28-12-10$ & 8500 & 150 & 2518 & 520 & 20.18 & 340 & 0.5 \\
\hline
\end{tabular}

Table 11: Strength of river sand (C25) stone dust (SDC25) and replacement of C25 with SDC 25 concrete at

\begin{tabular}{|c|c|c|c|c|c|c|c|c|c|}
\hline \multicolumn{10}{|c|}{ 28day } \\
\hline Sample No & $\begin{array}{l}\text { Date } \\
\text { Cast }\end{array}$ & $\begin{array}{l}\text { Date } \\
\text { crush }\end{array}$ & $\begin{array}{c}\text { Wt of } \\
\text { sample (g) }\end{array}$ & $\begin{array}{l}\text { Size of } \\
\text { sample } \\
(\mathrm{mm})\end{array}$ & \begin{tabular}{|l|} 
Density \\
$\mathrm{Kg} / \mathrm{m}^{3}$
\end{tabular} & \begin{tabular}{|c|} 
Crushing load \\
$(\mathrm{kg})$
\end{tabular} & $\begin{array}{l}\text { Strength } \\
\mathrm{n} / \mathrm{mm}^{2}\end{array}$ & $\begin{array}{c}\text { Cement } \\
\text { Content } \mathrm{kg} / \mathrm{m}^{3}\end{array}$ & $\begin{array}{l}\text { Free water } \\
\text { cement } \\
\text { ratio }\end{array}$ \\
\hline $\mathrm{C} 25 \mathrm{~A}$ & $\begin{array}{c}30-11- \\
10\end{array}$ & $\begin{array}{c}28-12- \\
10\end{array}$ & 8650 & 150 & 2563 & 500 & 20.54 & 360 & 0.5 \\
\hline C25B & $\begin{array}{c}30-11- \\
10\end{array}$ & $\begin{array}{c}28-12- \\
10\end{array}$ & 8550 & 150 & 2533 & 598 & 20.30 & 360 & 0.5 \\
\hline $\mathrm{C} 25 \mathrm{C}$ & $\begin{array}{c}30-11- \\
10\end{array}$ & $\begin{array}{c}28-12- \\
10\end{array}$ & 8550 & 150 & 2533 & 597 & 20.30 & 360 & 0.5 \\
\hline \multicolumn{10}{|c|}{ Average density $=2543$, Average strength $=20.38$} \\
\hline \multicolumn{10}{|c|}{ SDC 25} \\
\hline SDC25A & $\begin{array}{c}30-11- \\
10\end{array}$ & $\begin{array}{c}28-12- \\
10\end{array}$ & 8580 & 150 & 2542 & $630-$ & 20.37 & 360 & 0.5 \\
\hline SDC25B & $\begin{array}{c}3041- \\
10\end{array}$ & $\begin{array}{c}28-12- \\
10\end{array}$ & 8680 & 150 & 2571 & 590 & 20.28 & 360 & 0.5 \\
\hline SDC25C & $3 \mathrm{~d} 4 \mathrm{i} 4 \&$ & $\begin{array}{c}\text {,284\&- } \\
\text { W- }\end{array}$ & g63a. & 150 & 2561 & 620 & 20,54 & 360 & 0.5 \\
\hline \multicolumn{10}{|c|}{ Average density $=2549$, Average strength $=20.39$} \\
\hline \multicolumn{10}{|c|}{ Replacement of C 25 with SDC 25} \\
\hline
\end{tabular}


Ubi Stanley Emmanuel

\begin{tabular}{|l|c|c|c|c|c|c|c|c|c|}
\hline C20/SDC25A & $\begin{array}{c}30-11- \\
10\end{array}$ & $\begin{array}{c}28-12- \\
10\end{array}$ & 8580 & 150 & 2542 & 598 & 20.38 & 360 & 0.5 \\
\hline C20/SDC25B & $\begin{array}{c}30-11- \\
10\end{array}$ & 284240 & 8680 & 150 & 2571 & 600 & 20.71 & 360 & 0.5 \\
\hline $\begin{array}{l}\text { C20/SDC25C } \\
\begin{array}{c}30-11- \\
10\end{array}\end{array}$ & $2842-10$ & 8660 & 150 & 2565 & 598 & 2.057 & 360 & 0.5 \\
\hline
\end{tabular}

\subsection{DENSITY OF THE CONCRETE CUBES}

This can be expressed as the ration of weight to volume in mathematical form.

Density $\quad$ (D) $=\frac{\text { Weight }}{\text { Volume }}$

This is the factor that affect the strength of concrete. The higher the density the higher the compressive strength, as indicated in Table 12.

Stone dust has normal weight because their densities are comparable to that of sharp sand concrete according to Neville (2003) ranges from 2200 to $260 \mathrm{Qkg} / \mathrm{m}^{2}$. The result obtained was that density of quarry dust higher than that of sharp sand.

Table 12: The average density and strength for C20

\begin{tabular}{|c|c|c|c|}
\hline $\begin{array}{c}\text { CURING AGE } \\
\text { Days }\end{array}$ & $\begin{array}{c}\text { DENSITY C20 } \\
\left(\mathrm{Kg} / \mathrm{m}^{3}\right)\end{array}$ & $\begin{array}{c}\text { DENSITY } \\
\text { SDC20 } \\
\left(\mathrm{Kg} / \mathrm{m}^{3}\right)\end{array}$ & $\begin{array}{c}\text { DENSITY } \\
\mathrm{C} 20 / \mathrm{SDC} 20 \\
\left(\mathrm{Kg} / \mathrm{m}^{3}\right)\end{array}$ \\
\hline 7 & 2385 & 2410 & 2469 \\
\hline 14 & 2368 & 2368 & 2392 \\
\hline 21 & 2513 & 2916 & 2866 \\
\hline 28 & 2532 & 2570 & 2531 \\
\hline
\end{tabular}

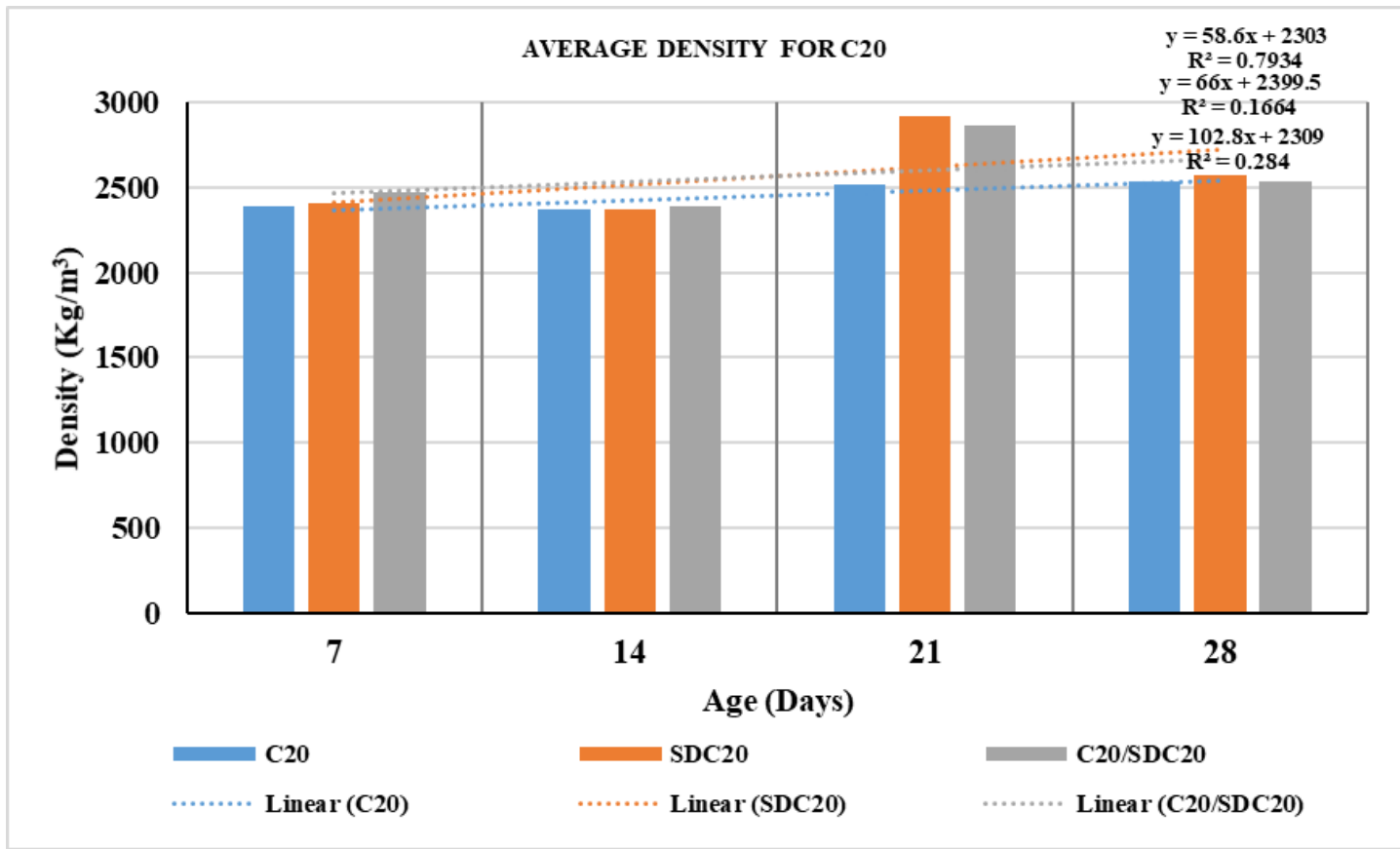

Figure 4: Average density for C20 
Effect of Replacing Sharp Sand with Stone Dust as Fine Aggregate in Concrete

Table 13: The average density and strength for $\mathrm{C} 25$

\begin{tabular}{|c|l|l|l|}
\hline 7 & 2424 & 2475 & 2452 \\
\hline 14 & 2331 & 2561 & 2417 \\
\hline 21 & 2328 & 2440 & 2485 \\
\hline 28 & 2543 & 2549 & 2551 \\
\hline
\end{tabular}

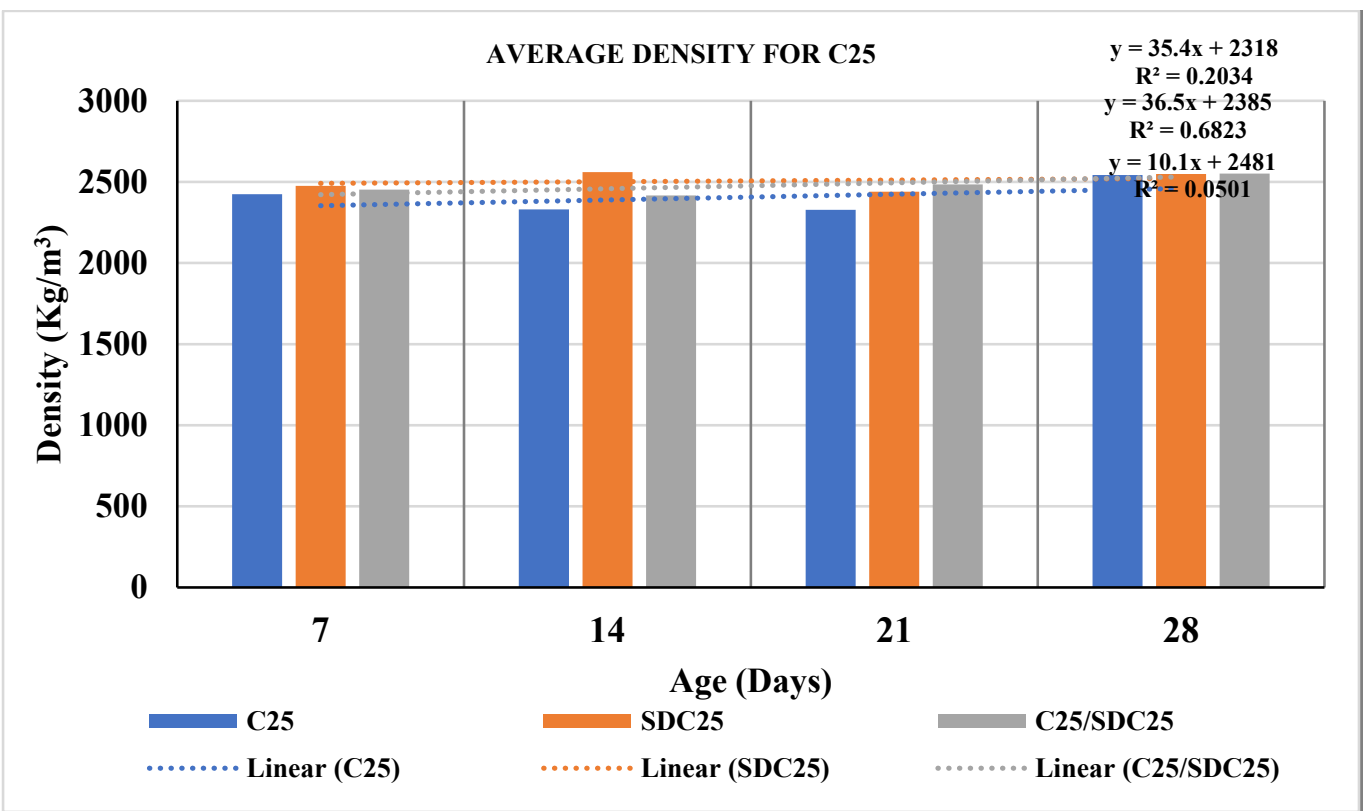

Figure 5: Average density for C25

Table 14: The average strength for $C 20$

\begin{tabular}{|c|c|c|c|}
\hline Curing Age & $\begin{array}{c}\text { Strength } \\
\text { C20 } \\
\left(\mathrm{N} / \mathrm{mm}^{2}\right)\end{array}$ & $\begin{array}{c}\text { Strength } \\
\text { SDC20 } \\
\left(\mathrm{N} / \mathrm{mm}^{2}\right)\end{array}$ & $\begin{array}{c}\text { Strength with } \\
\text { SDC20 } \\
\left(\mathrm{N} / \mathrm{mm}^{2}\right)\end{array}$ \\
\hline 7 & 19.12 & 19.32 & 19.17 \\
\hline 14 & 18,98 & 18.98 & 19.17 \\
\hline 21 & 20.14 & 20.77 & 20.41 \\
\hline 28 & 20.29 & 20.60 & 20.28 \\
\hline
\end{tabular}

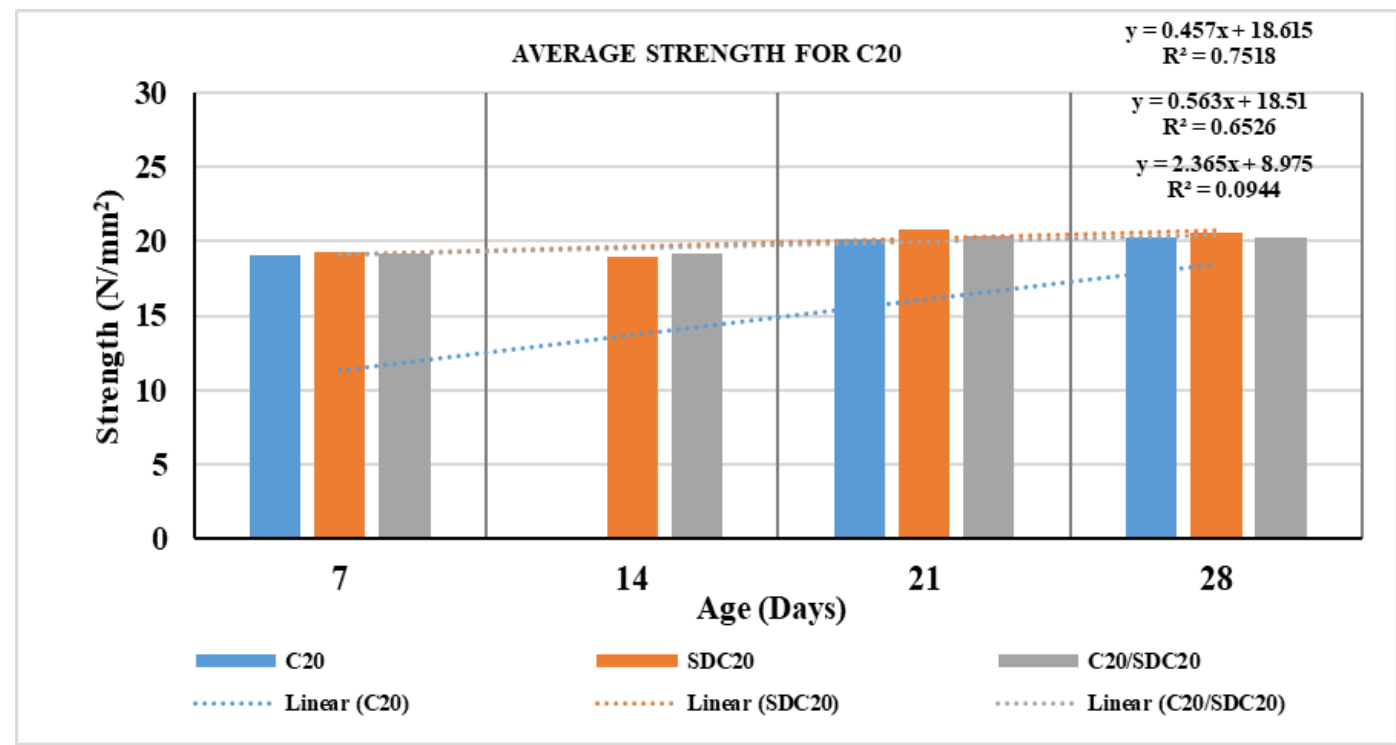

Figure 6: Average strength for C20 
Table 15: The average strength for C25

\begin{tabular}{|c|c|c|c|}
\hline 7 & 19.43 & 19.84 & 19,65 \\
\hline 14 & 18.69 & 20.49 & 19.37 \\
\hline 21 & 18.65 & 19.08 & 19.92 \\
\hline 28 & 20.38 & 20.39 & 20.55 \\
\hline
\end{tabular}

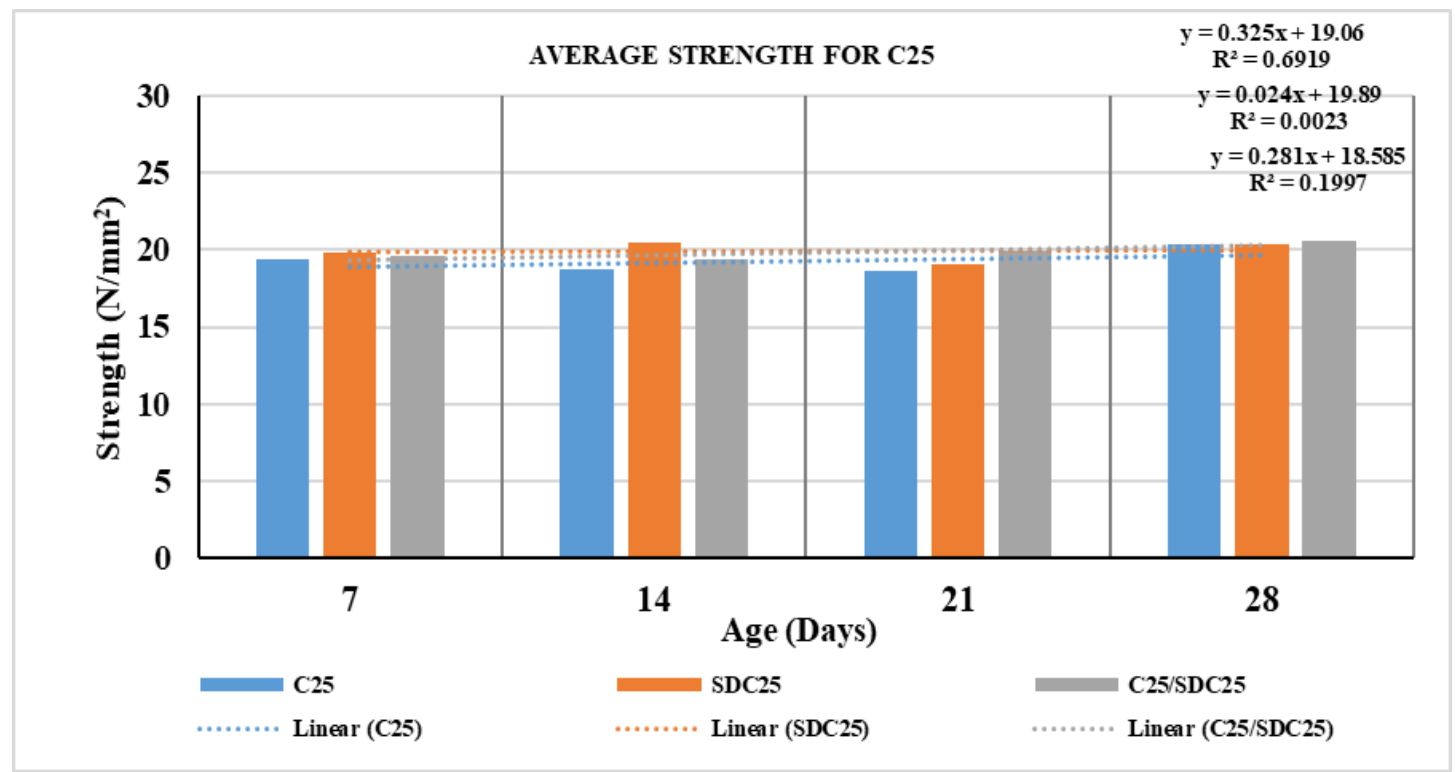

Figure 7: Average strength for C25

\subsection{STANDARD DEVIATION}

The account of the deviation of every value from the distribution has been taking by using the standard deviation. The value which is higher shown that there was a high level of consistencies during the laboratory work.

Standard deviation $(\mathrm{SD})=\frac{\sqrt{\sum}(x-x) 2}{n}$

Where $\mathrm{xi}=\mathrm{x}_{1}, \mathrm{x}_{2}, \mathrm{x}_{3}$, the value of the compressive strength for the cube produced.

$\mathrm{x}=$ mean of number of value

$\mathrm{n} \quad=$ Total number of value

The standard deviation for the various mixes designed are to be calculated at 7, 14, 21 and 28 days to know the strength.

For $\mathrm{C}_{2} \mathrm{O}$ at 7 days curing strength

$$
S D=\frac{(18.88-19.12)^{2}+(19.03-19.12)^{2}+(19.47-19.12)^{2}}{274^{3} \mathrm{~N} / \mathrm{mm}^{2}}
$$

For $\mathrm{SDC}_{2} \mathrm{O} 7$ days strength

$$
\begin{aligned}
& S D^{2}=\frac{(19.52-19.32)^{2}+(19.59-19.32)^{2}+(18.88-19.32)^{2}}{3} \\
& =0.105 \mathrm{~h} / \mathrm{MM}
\end{aligned}
$$


For $\mathrm{C}_{2} 0$ at 14 days 1 strength

$$
\begin{aligned}
& S D^{2}=\frac{(18.79-18.98)^{2}+(18.98)^{2}+(19.07-18.98)^{2}=(19.09-18.93)^{2}}{3} \\
& 0.102 \mathrm{~N} / \mathrm{mm}^{2}
\end{aligned}
$$

For $\mathrm{C}_{2} \mathrm{O}$ at 14 days 1 strength

$$
\begin{aligned}
& S D^{2}=\frac{(18.79-18.98)^{2}+(19.07-18.98)^{2}+(19.09-18.98)^{2}}{3} \\
& 2.7 \mathrm{~N} / \mathrm{mm}^{2}
\end{aligned}
$$

For SOCO20 at 14 days strength

$$
\begin{aligned}
& S D^{2}=\frac{(19.09-18.98)^{2}+(18.79-18.98)^{2}+(19.07-18.98)^{2}}{3} \\
& 2.7 \mathrm{~N} / \mathrm{mm}^{2}
\end{aligned}
$$

For $\mathrm{X}_{2} \mathrm{O}$ with $\mathrm{SDC}_{2} \mathrm{O}$ at 14 days strength

$\mathrm{SD}^{2}(19.50-19.17)^{2}=+(18.56-19.17)^{2}+(19.47-19.17)^{2}$

$0.19 \mathrm{~N} / \mathrm{mm}^{2}$

For $\mathrm{C}_{2} \mathrm{O}$ at 21 days strength

$$
\begin{aligned}
& S D^{2}=\frac{(19.06-20.14)^{2}+(21.10-20.14)^{2}+(20.28-20.14)^{2}}{3} \\
& 0.702 \mathrm{~N} / \mathrm{mm}^{2}
\end{aligned}
$$

For $\mathrm{SDC}_{2} \mathrm{O} 21$ days strength

$$
\begin{aligned}
& S D^{2}=\frac{(20.77-20.77)^{2}+(20.20-20.77)^{2}+(21.35-2.77)^{2}}{3} \\
& =0.105 n / M M
\end{aligned}
$$

For $\mathrm{C}_{2} 0$ at 14 days 1 strength

$$
\begin{aligned}
& S D^{2}=\frac{(18.79-18.98)^{2}+(18.98)^{2}+(19.07-18.98)^{2}=(19.09-18.93)^{2}}{3} \\
& 0.2204 \mathrm{~N} / \mathrm{mm}^{2}
\end{aligned}
$$

For $\mathrm{C}_{2} \mathrm{O}$ with $\mathrm{SDC}_{2} \mathrm{O} 21$ days strength

$$
\begin{aligned}
& S D^{2}=\frac{(19.47-20.41)^{2}+(21.36-20.41)^{2}+(20.42-20.41)^{2}}{3} \\
& =3.008 \mathrm{~N} / \mathrm{mm}^{2}
\end{aligned}
$$


For $\mathrm{C}_{2} \mathrm{O}$ at 28 days strength

$$
\begin{aligned}
& S D^{2}=\frac{(20.37-20.29)^{2}+(20.49-20.29)^{2}+(20.02-20.29)^{2}}{3} \\
& =02.436 \mathrm{~N} / \mathrm{mm}^{2}
\end{aligned}
$$

For sdc25 at 7 days strength

$$
\begin{aligned}
& S D^{2}=\frac{(20.29-19.84)^{2}+(19.59-19.84)^{2}+(19.64-19.84)^{2}}{3} \\
& =0101 \mathrm{~N} / \mathrm{mm}^{2}
\end{aligned}
$$

For C25 with SOC25 at 27 days strength

$$
S D^{2}=\frac{(17.88-19.56)^{2}+(20.42-1965)^{2}+(20.66-19.65)^{2}}{3}
$$

1.58.19N/mm $\mathrm{mm}^{2}$

For C25 at 14 days strength

$$
\begin{aligned}
& S D^{2}=\frac{(19.31-18.69)^{2}+(18.12-18.69)^{2}+(18.64-18.69)^{2}}{3} \\
& =1.069 \mathrm{~N} / \mathrm{mm}^{2}
\end{aligned}
$$

For SDC25 at 14 days strength

$$
\begin{aligned}
& S D^{2}=\frac{(21.25-20.49)^{2}+(21.01-20.49)^{2}+(19.22-20.49)^{2}}{3} \\
& 0.8203 \mathrm{~N} / \mathrm{mm}^{2}
\end{aligned}
$$

For C25 with SDC25 at 14 days strength

$$
\begin{aligned}
& S D^{2}=\frac{(18.95-19.37)^{2}+(21.09-19.37)^{2}+(28.09-19.37)^{2}}{3} \\
& =1.8548 \mathrm{~N} / \mathrm{mm}^{2}
\end{aligned}
$$

For C25 at 21 days strength

$$
\begin{aligned}
& S D^{2}=\frac{(18.60-18.65)^{2}+(19.23-18.63)^{2}+(18.14-18.65)^{2}}{3} \\
& =1.0400 \mathrm{~N} / \mathrm{mm}^{2}
\end{aligned}
$$

For SDC25 at 21 days strength

$$
\begin{aligned}
& S D^{2}=\frac{(19.86-19.08)^{2}+(18.73-19.08)^{2}+(20.81-19.08)^{2}}{3} \\
& =0.2412 \mathrm{~N} / \mathrm{mm}^{2}
\end{aligned}
$$


For C25 with SOC25 at 21 days strength

$$
\begin{aligned}
& S D^{2}=\frac{(20.28-19.92)^{2}+(18.39-19.92)^{2}}{3} \\
& 1.2876 \mathrm{~N} / \mathrm{mm}^{2}
\end{aligned}
$$

For x25 at 28 days strength

$$
\begin{aligned}
& S D^{2}=\frac{(20.54-20.38)^{2}+(20.38-20.30)^{2}+(20.30-20.38)^{2}}{3} \\
& =0.0384 \mathrm{~N} / \mathrm{mm}^{2}
\end{aligned}
$$

For SDC25 at 28 days strength

$$
\begin{aligned}
& S D^{2}=\frac{(20.37-20.39)^{2}+(20.28-20.39)^{2}+(20.54-20.39)^{2}}{3} \\
& 1.3448 \mathrm{~N} / \mathrm{mm}^{2}
\end{aligned}
$$

For C25 with SDC25 at 14 days strength

$$
\begin{aligned}
& S D^{2}=\frac{(18.95-19.37)^{2}+(21.09-19.37)^{2}+(28.09-19.37)^{2}}{3} \\
& =1.8548 \mathrm{~N} / \mathrm{mm}^{2}
\end{aligned}
$$

For C25 with SDC25 at 28 days strength

$$
S D^{2}=\frac{(20.38-20.55)^{2}+(20.71-20.55)^{2}+(20.57-20.55)^{2}}{3}
$$

\subsection{TARGET MEAN STRENGTH}

Variation of concrete in production, is the amount by which the produced concrete strength is greater than the characteristic strength. Margin for design takes care of the variation of concrete, in production, it is the amount by which the produced concrete strength is greater than the characteristic strength. From the results shown in Table 16 and figure 8, comparative analysis of the concrete produced replacing stone dust with sharp sand in each case was examined. The formula used are:

$\mathrm{FM}=\mathrm{fe}+$ Ks where

$\mathrm{Fm}=\quad$ the target mean strength

$\mathrm{Fe}=\quad$ specified characteristic strength

$\mathrm{Ks}=\quad$ the margin, which is the product of

$\mathrm{S}=$ standard deviation and

$\mathrm{K}=$ a constant

The $\mathrm{K}$ is device from the mathematics of the normal distribution and increase as the proportion of detective is decrease thus:

$\mathrm{K}$ for $=$ For 10 percentage defective $=1.28$

$\mathrm{K}$ for 5 percentage defective $=1.64$

$\mathrm{K}$ for $\mathrm{M}$ percentage defective $=2.33$.

A value of 5\% defective is however, permitted in cp 110, 1985 for instance; the target mean strength of gate 20 concrete is $\mathrm{Fm}=\mathrm{fe}+\mathrm{ks}$

$=20+(1.64 \times 8)=33 \mathrm{~N} / \mathrm{mm}^{2}$. 
Ubi Stanley Emmanuel

Table 16: Target mean strength

\begin{tabular}{|c|c|c|}
\hline Age day & Strength $\left(\mathrm{N} / \mathrm{mm}^{2}\right)$ & Target mean strength \\
\hline 7 & C20:19.12 & $57 \%$ \\
\hline 14 & C20:18.98 & $62 \%$ \\
\hline 21 & C20;20.14 & $64 \%$ \\
\hline 28 & C20:20.29 & 68 \\
\hline \multicolumn{3}{|c|}{} \\
\hline 7 & SDC20: 19.32 & $72 \%$ \\
\hline 14 & SDC20;18.98 & $66 \%$ \\
\hline 21 & SDC20:2,14 & $74 \%$ \\
\hline 28 & SDC20: 20.60 & $77 \%$ \\
\hline \multicolumn{3}{|c|}{} \\
\hline \multicolumn{3}{|c|}{ Target mean strength } \\
\hline 7 & C20/SDC20: 19.79 & $58 \%$ \\
\hline 14 & C20/SDC20: 19.17 & $60 \%$ \\
\hline 21 & C20/SDC20: 20.41 & $68 \%$ \\
\hline \multicolumn{3}{|c|}{} \\
\hline 7 & C25:19.43 & $54 \%$ \\
\hline 14 & C25: 18.69 & $60 \%$ \\
\hline 21 & C25: 18.65 & $64 \%$ \\
\hline 28 & C25: 20.38 & $70 \%$ \\
\hline
\end{tabular}

Target mean, strength

\begin{tabular}{|c|c|c|}
\hline 7 & C25/SDC25: $19: 65$ & $56 \%$ \\
\hline 14 & C25/SDC 25: 19.37 & $62 \%$ \\
\hline 21 & C25/SDC $25: 19.92$ & $68 \%$ \\
\hline 28 & C25/SDC 25: 20.55 & $72 \%$ \\
\hline
\end{tabular}

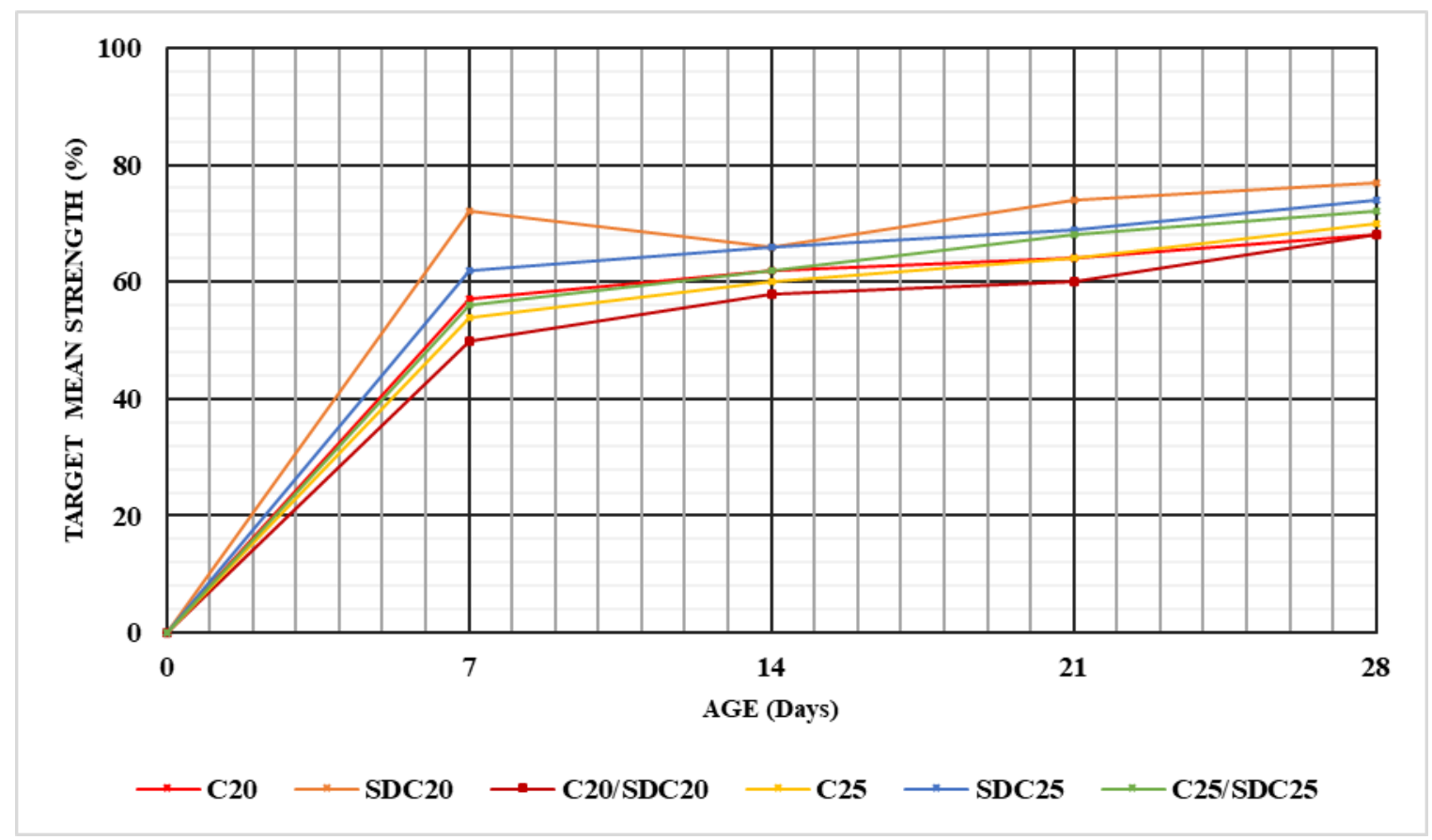

Figure 8: Target mean strength gradient 


\section{CONCLUSION AND RECOMMENDATION}

From the research work carried out on the effect of replacing sharp sand with stone dust in concrete work, many inferences have been deduced from the results of the investigation, some of the conclusion based on this investigation are summarized thus:

1) If the proper design procedures are followed, stones dust as fine aggregate does not have any detrimental effect on the overall properties of concrete, rather it helps to improve on the properties, if sharp sand could be added with stone dust it will help the concrete to be detrimental.

2) Sharp sand with stone dust concrete develop $6-12 \%$ more compressive strength than sharp sand (fine aggregate) while stone dust concrete develops 6 -14\% more compressive strength also than river sand, it attains higher strength at early age than sharp sand concrete.

3) The cost implication replacing stone dust with sharp sand in concrete is within the acceptable margin of price differentials. Economical to consider replacing sharp sand with stone dust for use when a cheaper means of transportation is devised or the construction site is closer to the site were the material can be obtained.

4) Concrete with replacement requires more water than river sand concrete that's why it's develops higher strength. In this case, when replacing sharp sand with stone dust, in concrete, you must know that enough water is supplied to the concrete.

\subsection{RECOMMENDATION}

In view of the foregoing engineering advantages of replacing sharp sand with stone dust in concrete, the following recommendations are presented to stakeholders:

1) In construction industry professionals should come up with more information regarding the replacing of sharp sand with stone dust in concrete by investing in research into replacing and how it can be used to obtain concrete with must higher strength than that form river sand or that of stone dust

2) Government should make the transportation section more efficient so as to reduce the cost of transporting good and materials for construction and other constitutions of concrete from one place to another.

3) Awareness campaigns should be organized by professional in Civil Engineering way of seminar and workshop to enlighten the general public on the cost and strength consideration for replacing sharp sand with stone dust in concrete work, by so doing more and more people will come to embrace the replacing of sharp sand with stone dust instead of using only sharp sand in the construction site they will use both the sharp sand with stone dust. By so doing stone dust will give more strength together with the strength of river sand and the strength will be higher than that of sharp sand only. For that, many people will like to use both the sharp and with stone dust for the construction of their own structures.

\section{SOURCES OF FUNDING}

This research received no specific grant from any funding agency in the public, commercial, or not-for-profit sectors.

\section{CONFLICT OF INTEREST}

The author have declared that no competing interests exist.

\section{ACKNOWLEDGMENT}

None. 


\section{REFERENCES}

[1] Agbede, A. J. (2002) Suitability of Quarry Dust as Partial Replacement for sand in Hollow Block, Nse Technical Journal, Vol. 46, No., pp22 -28.

[2] British Standard Institution BS 882, Part 2: 1973. Coarse and Fine aggregate from National Sources, London.

[3] Ducatz, E. L. (1995) Effective use of Aggregate Fine ICAR $3^{\text {rd }}$ Annual Symposium.

[4] Fowler, D. W. and Constantino, G. A. (1997) International Research on Firms in Concrete, ICAR $5^{\text {th }}$ Annual Symposium.

[5] Nagaraji, T. S. and Zahida B. (1999), Efficient Utilization of Rock Dust and Pebbles as Aggregate in Portland Cement Concrete, The Indian Concrete Journal, Vol 70, No. 1, pp 1-4.

[6] Neville, A. M. (2003). Properties of Concrete 4thEdn. Pearson Education.

[7] Owoiabi, A. O. (1996), Use of Locally Available Fine and Coarse aggregate, Using Laterite as an Admixture, Nse Technical Journal, Vol. No. 5, pp. 45 -47.

[8] Pofale, A. D. and Kulkarni, S. S. (1998) Comparative Study of Strength Properties of Concrete Mixes with Natural Sand replaced fully or partially be crushes stone powder (Basalt) from aggregate crushing waste, National Seminar on Advances on special concretes, Indian Concrete institute, Bang, Love, Indian, pp 227 240.

[9] Prakash, D. S. and Kumar, V. C. (2004), Investigations on Concrete using Stone waste as fine Aggregate for Concrete. The Indian concrete Journal, Vol. 7p. 45 - 49.

[10] Ubi S. E., Nkra P.O., Agbor R.B., Ewa D.E. and Nuchal M (2020). Efficacy of basalt and granite as coarse aggregate in concrete mixture, International Journal of Engineering Technologies and Management Research, 7(9): 1-9. 